\title{
Observing an intermediate-mass black hole GW190521 with minimal assumptions
}

\author{
Marek Szczepańczyk®, ${ }^{1}$ Sergey Klimenko®, ${ }^{1}$ Brendan O’Brien $\odot,{ }^{1}$ Imre Bartos $\odot,{ }^{1}$ V. Gayathri®, ${ }^{1}$ \\ Guenakh Mitselmakher®, ${ }^{1}$ Giovanni Prodi $\odot{ }^{2,3}$ Gabriele Vedovato $\odot,{ }^{4,5}$ Claudia Lazzaro® ${ }^{4,5}$ Edoardo Milotti®, ${ }^{6,7}$ \\ Francesco Salemi $\odot,{ }^{2}$ Marco Drago $\odot,{ }^{8,9}$ and Shubhanshu Tiwari $\oplus^{10,2,3}$ \\ ${ }^{1}$ University of Florida, Gainesville, Florida 32611, USA \\ ${ }^{2}$ Università di Trento, Dipartimento di Fisica, I-38123 Povo, Trento, Italy \\ ${ }^{3}$ INFN, Trento Institute for Fundamental Physics and Applications, I-38123 Povo, Trento, Italy \\ ${ }^{4}$ Università di Padova, Dipartimento di Fisica e Astronomia, I-35131 Padova, Italy \\ ${ }^{5}$ INFN, Sezione di Padova, I-35131 Padova, Italy \\ ${ }^{6}$ Dipartimento di Fisica, Università di Trieste, I-34127 Trieste, Italy \\ ${ }^{7}$ INFN, Sezione di Trieste, I-34127 Trieste, Italy \\ ${ }^{8}$ Università di Roma La Sapienza, I-00185 Roma, Italy \\ ${ }^{9}$ INFN, Sezione di Roma, I-00185 Roma, Italy \\ ${ }^{10}$ Physik-Institut, University of Zurich, Winterthurerstrasse 190, 8057 Zurich, Switzerland
}

(Received 26 September 2020; accepted 3 March 2021; published 27 April 2021)

\begin{abstract}
On May 21, 2019 the Advanced LIGO and Advanced Virgo detectors observed a gravitationalwave transient GW190521, the heaviest binary black-hole merger detected to date with remnant mass of $142 M_{\odot}$ that was published recently. This observation is the first strong evidence for the existence of intermediate-mass black holes. The significance of this observation was determined by the coherent WAVEBURST (cWB) search algorithm, which identified GW190521 with minimal assumptions of its source model. In this paper, we show the performance of cWB for the detection of the binary black-hole mergers without use of the signal templates, describe the details of the GW190521 detection, and establish the consistency of the model-agnostic reconstruction of GW190521 by cWB with the theoretical waveform model of a binary black hole.
\end{abstract}

DOI: 10.1103/PhysRevD.103.082002

\section{INTRODUCTION}

The third observing run (O3) of the Advanced LIGO [1] and Advanced Virgo [2] network has brought discoveries of new binary sources [3-5], together with a wealth of gravitational-wave (GW) detection candidates [6]. The GW190521 [5] signal observed during the first half of the O3 Advanced LIGO run has an estimated remnant mass of $142 M_{\odot}$ making it the most massive black hole found through GWs to date. GW190521 [5] provides strong observational evidence for the existence of intermediatemass black holes (IMBHs) that are usually defined as black holes with mass in the range $10^{2}-10^{5} M_{\odot}[7,8]$.

Following the first observation of a binary black-hole (BBH) merger [9], the first two observing runs of Advanced LIGO and Virgo [10] have revealed a population of BBHs with component masses up to $50 M_{\odot}$ and remnant black-hole mass up to $85 M_{\odot}$ [10]. These observations are consistent with a mechanism known as pair-instability supernova (PISN) [11-14], which prevents the formation of heavier black holes from stellar core collapse. Stars with a helium core mass in the range 64-135 $M_{\odot}$ (PISN mass gap) undergo pulsational pair instability and leave no remnant. Stars with core mass above $135 M_{\odot}$ are thought to directly collapse to IMBHs. For GW190521 the estimated masses of the component black holes are $85_{-14}^{+21}$ and $65_{-18}^{+17} M_{\odot}$ suggesting that the primary black hole may be well inside the PISN mass gap. Observations of such IMBH binary sources start to probe the boundaries of the PISN mass gap and may reveal the $\mathrm{BBH}$ formation mechanisms outside of the stellar evolution.

Because of their high mass, the IMBH binary systems are expected to merge at frequencies below $100 \mathrm{~Hz}$, while detector sensitivity is limited by seismic noise below $20 \mathrm{~Hz}$. As a result, the detectors are mostly sensitive to the gravitational waves emitted at the final stages of the binary evolution, merger, and ringdown. For IMBH binary systems, the observed GW signal is short in duration and challenging to detect and reconstruct by template-based algorithms. The excess power cWB [15] is a templateindependent search algorithm that uses minimal assumptions of the signal model to detect GWs. It does not depend on detailed waveform features such as the higher-order modes, high-mass ratios, misaligned spins, eccentric orbits, and possible deviations from general relativity, and it operates even in cases where the lack of reliable models 


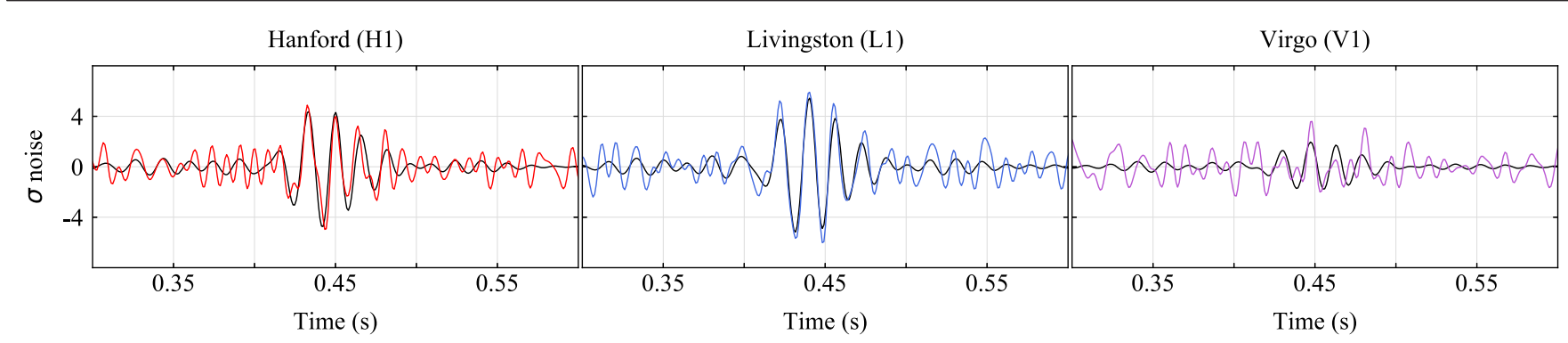

FIG. 1. GW190521 observed by the H1 (left panel), L1 (middle panel), and V1 (right panel) detectors. Times are shown relative to May 21, 2019 at 03:02:29 UTC. Color curves: the detector time series are filtered with a $28128 \mathrm{~Hz}$ band-pass filter and cleaned to remove the $60 \mathrm{~Hz}$ power line. Black curves: signal reconstruction by cWB. Visually, the cWB reconstruction is in agreement with the amplitudes around GW190521.

poses limitations for matched filtering methods. Therefore, cWB is suitable for detecting sources where reliable templates are not readily available $[16,17]$.

Prior to the discovery of GW190521, LIGO and Virgo performed several searches that allowed them to constrain the rate of IMBH sources, prepare the detection algorithms, and improve the IMBH signal models. The first searches for gravitational waves from IMBH binaries were carried out with cWB on the data from the initial LIGO and Virgo observing runs (2005-2010) [18,19]. A search for perturbed IMBH remnants was performed on the same data with the ringdown template search [20]. Later, the IMBH searches were conducted with $\mathrm{O} 1$ and $\mathrm{O} 2$ data both by $\mathrm{cWB}$ and the full inspiral-merger-ringdown template pipelines [21,22]. The IMBH binary searches carried out on the $\mathrm{O} 1$ and $\mathrm{O} 2$ data produced the most stringent upper limit of $0.2 \mathrm{Gpc}^{-3} \mathrm{yr}^{-1}$ on the IMBH merger rates [21]. Improvements of the advanced detector sensitivities in the O3 run [23] tripled the IMBH search volume resulting in the observation of the first IMBH binary event GW190521 $[5,24]$ identified by the cWB search with high confidence. Together with the first ever GW detection GW150914 [9] and the heaviest BBH system GW170729 in the O1 and O2 data [10], the GW190521 is yet another demonstration of cWB to detect unexpected binary sources.

In this paper, we demonstrate the capability of cWB to detect GW190521 with high confidence without the use of templates. We show that the cWB reconstruction of GW190521 is in agreement with the waveforms derived from the model-based Bayesian LALInference [25] analysis used for inference of the physical properties of the source. The main results of the cWB analysis are presented in the discovery [5] and astrophysical implication [24] papers. In Sec. II, we describe the details of the GW190521 observation with $\mathrm{cWB}$, including the low-latency analysis and the GW190521 sky localization and describe the detection significance established by cWB. In Sec. III, we show consistency between the cWB waveform reconstruction and the best fits of the Bayesian inference waveforms from LALInference. We demonstrate and quantify that the cWB reconstruction is more consistent with waveforms from precessing BBHs including higher-order modes rather than binaries lacking these effects. Appendixes A-C outline the cWB searches in $\mathrm{O} 3$ and the detection procedure.

\section{OBSERVATION}

\section{A. Online detection and sky localization}

GW190521 triggered an initial alert [26] within minutes, and the event was detected by template-based PyCBC [27] and morphology-independent cWB [15] pipelines with false-alarm rates (FARs) 1 per 8.3 years and lower than 1 per 28 years, respectively. The online cWB FAR was estimated using one day of coincident data around the triggered time. By combining all previously accumulated background data from the low-latency analysis, the GW190521 FAR was estimated to be lower than 1 per 500 years.

The waveform reconstructed by cWB in the low-latency analysis (see Figs. 1 and 2) did not show a typical chirping signal usually expected for BBH signals typically detected by LIGO and Virgo. Instead, GW190521 was a short signal with a duration of $0.1 \mathrm{~s}$ and less than four cycles in the frequency band $30-80 \mathrm{~Hz}$ with almost symmetric shape. Such a signal morphology is typical for the high-mass BBH events when the system merges at low frequency where the sensitivity of the detectors is affected by seismic noise. Assuming that the binary merges at the peak signal frequency of $58 \mathrm{~Hz}$ (twice the orbital frequency), the total mass of the system in the detector frame should exceed $300 M_{\odot}$ for equal mass $\mathrm{BH}$ components. The most plausible explanation for this signal is the merger of a binary $\mathrm{BH}$ system where the remnant is an intermediatemass black hole (other source interpretations can be found in [24]).

In the low-latency analysis, cWB estimated the sky area to be $1474 \mathrm{deg}^{2}$ (90\% confidence interval). It is in agreement with $1163 \mathrm{deg}^{2}$ (90\% credible interval) reported initially with the public alert [26] and computed with the Bayestar [28] sky localization algorithm using BBH templates [29]. In the follow-up analysis, the sky maps were computed using calibrated data resulting in 1653 and $765 \mathrm{deg}^{2}$ [24] for cWB 


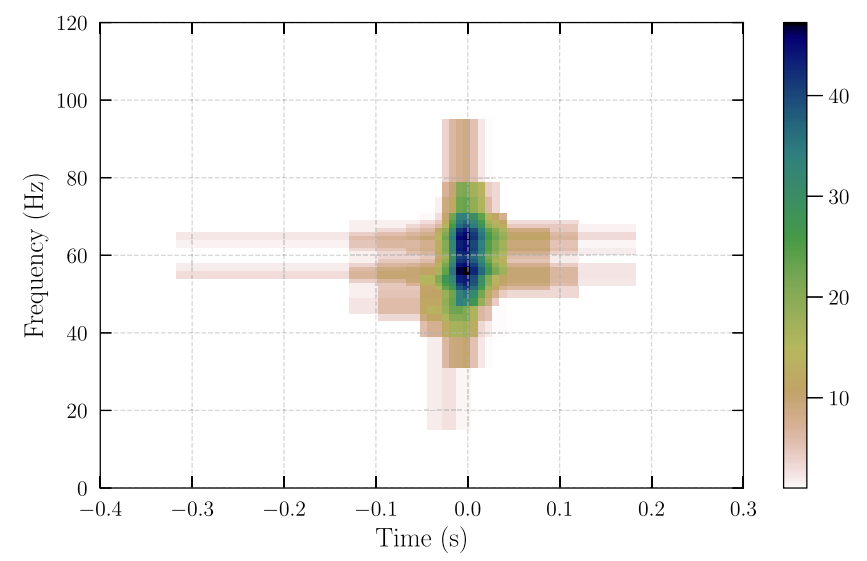

FIG. 2. Time-frequency representation of GW190521 with a superposition of wavelet pixels selected by cWB. The pixel magnitudes are the squared network signal-to-noise ratio.

and Bayestar, respectively. Figure 3 compares the initially released sky maps with those computed by cWB for the LIGO Hanford, LIGO Livingston, Virgo (HLV) detector network.

\section{B. Detection significance}

The offline cWB analysis was conducted with the HL (LIGO Hanford and LIGO Livingston) and HLV detector networks. Because of the difference in sensitivity between the LIGO and Virgo detectors and larger nonstationary noise in Virgo [23], the HL network was used to determine the detection significance of the GW190521 event, while the HLV network was used for the waveform reconstruction described in Sec. III. As described in [5], the data from the $\mathrm{GW}$ detectors were conditioned prior to further analysis. Specifically for the cWB analysis, we additionally mitigated the nonstationary anthropogenic noise (see Appendix B).

The detection significance of GW190521 was determined by time shifting the Hanford detector data with

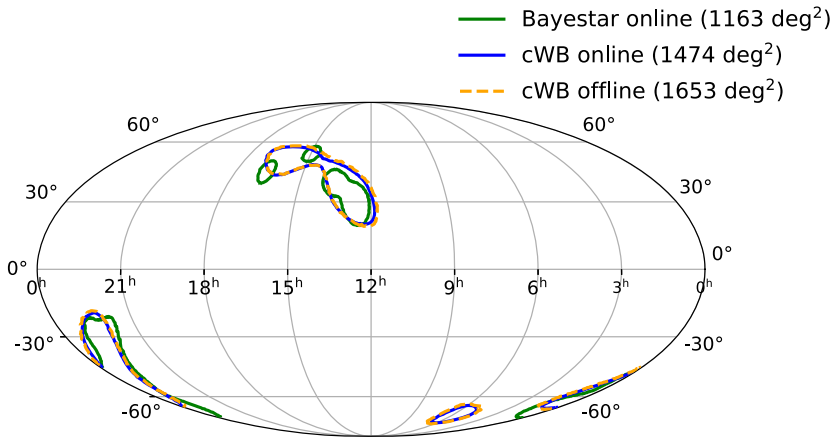

FIG. 3. GW19052190\% credible areas source localization with the three-detector LIGO-Virgo network reported by the lowlatency analysis from Bayestar by using BBH templates [29] and cWB sky localization without templates for low-latency and offline analyses. respect to the Livingston detector data to accumulate events of nonastrophysical origin. The time shifts were selected to be much longer ( $1 \mathrm{~s}$ or more) than the expected signal time delay between the detectors ( $2 \mathrm{~ms}$ for GW190521). To perform the background analysis, we used 9.4 days of coincident data around the event inside the GPS time interval [1241011102, 1242485126]. By using multiple time shifts, we accumulated an equivalent of 9800 years of background data.

Figure 4 presents the background distribution of timeshifted data and GW190521. Only two events have the cWB detection statistic $\eta_{c}$ higher than the GW190521 event, both consistent with random coincidences of shortduration $(\approx 1$ cycle $)$ glitches observed in the LIGO frequency band $20-100 \mathrm{~Hz}$ (more information about noise sources can be found in Appendix B). The amount of the background and the number of louder events results in a false-alarm rate of 1 per 4900 years for GW190521, which constitutes a confident detection.

\section{Search sensitivity}

The detection range of IMBH sources has been increasing over time [18-22], mainly due to improvements in the low-frequency regime of the GW detectors and the algorithms used. To calculate the detection ranges for events similar to GW190521, we assume the NRsur7dq4 signal model [30] described in the next section. For the Advanced LIGO and Advanced Virgo, they are 1.1, 1.2, and $1.7 \mathrm{Gpc}$ for $\mathrm{O} 1, \mathrm{O} 2$, and the first half of $\mathrm{O} 3$ observing runs, respectively [24]. At present, the total search time volume for events like GW190521 is $9.1 \mathrm{Gpc}^{3} \mathrm{yr}$ [24], which is the sum of the $\mathrm{O} 1-\mathrm{O} 2$ contribution of $3.2 \mathrm{Gpc}^{3} \mathrm{yr}$ and of the nearly twice as large contribution from the first half of the $\mathrm{O} 3$ run: $5.9 \mathrm{Gpc}^{3} \mathrm{yr}$.

The rate of the IMBH binary mergers was initially established using the 2005-2007 dataset [18], and later it was constrained to $0.2 \mathrm{Gpc}^{-3} \mathrm{yr}^{-1}$ with data from the first two Advanced LIGO-Virgo observing runs [21]. In [24],

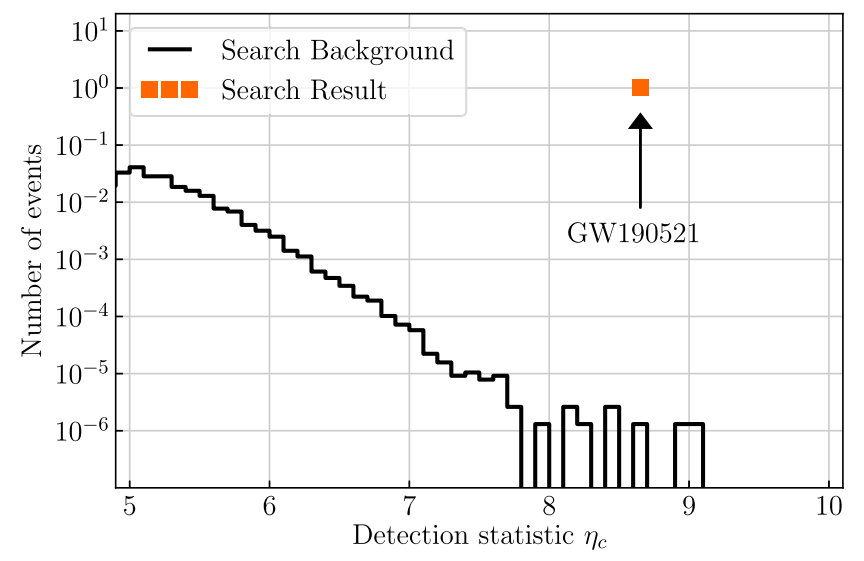

FIG. 4. The search background that established the false-alarm rate of GW190521 to be 1 per 4900 years. 


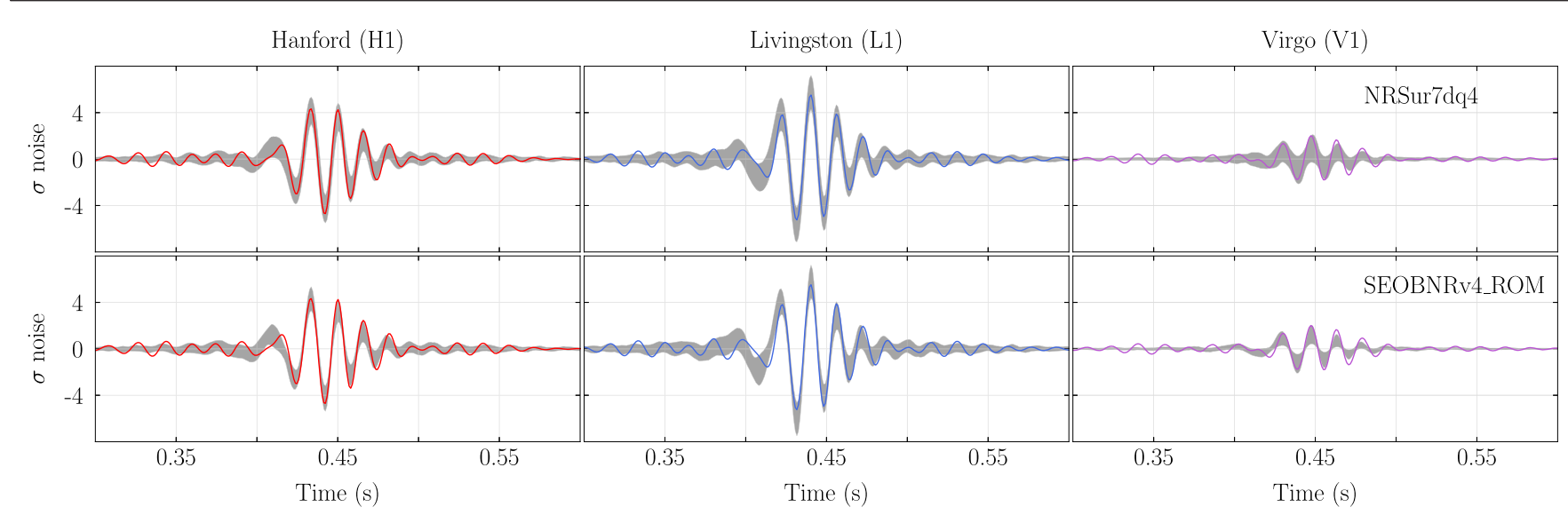

FIG. 5. Visualization of the consistency of the signal-agnostic measurement by cWB with two alternative models in the time domain: upper panel, NRSur7dq4 model; lower panel, SEOBNRV4_ROM model. The colored curves are the whitened waveform for GW190521 as reconstructed by cWB for the LIGO Hanford (red), LIGO Livingston (blue), and Virgo (violet); they are a direct measurement and do not change from the upper to the lower panels. The shaded belts are $90 \%$ confidence intervals per time sample at each detector and are model dependent.

the rate of events is shown to be $0.13_{-0.11}^{+0.3} \mathrm{Gpc}^{-3} \mathrm{yr}^{-1}$ [24], which is below the previously derived upper limits.

\section{WAVEFORM ANALYSIS}

In this section, we present the comparison of the GW190521 reconstructions with the cWB and Bayesian methods. In [24], the Bayesian reconstruction was carried out using the model-based LALInference [25,31], RIFT [32], and BAYESWAVE [33]. We perform the comparison with the model-based LALInference, and the purpose is to determine if the cWB and LALInference reconstructions are consistent among different signal models. We focus on the LALInference posterior distribution, but this method can be applied to other Bayesian reconstructions as well.

The cWB waveforms are derived directly from the data in the wavelet (time-frequency) domain [34] by selecting the excess power data samples above the average fluctuations of the detector noise. The cWB point estimate of the signal waveform in each detector is constructed as a linear combination of selected wavelets with the amplitudes defined by the constrained maximum likelihood method [15]. Similar to the band-pass filter shown in Fig. 1, cWB performs the time-frequency filtering of data. Such signalagnostic reconstruction may capture significant features of the signal that may not be well described by the model approximations and help to identify the limitations of the models used for Bayesian inference of GW190521. On the other hand, due to the excess power threshold, the low SNR wavelet components could be excluded from the cWB analysis resulting in a partial reconstruction of the GW190521 signal. In addition, the non-Gaussian noise fluctuations may bias the cWB reconstruction and add systematic errors not accounted for by the Bayesian inference. Therefore, a direct comparison of the cWB reconstruction, or any other excess power algorithm, with the best matching LALInference waveforms is not straightforward and requires a more accurate statistical treatment as described below.

In this analysis, we compare cWB point estimate waveform with signals from the inspiral-merger-ringdown SEOBNRV4_ROM [35,36] that is based on the effectiveone-body formalism, and the numerical relativity surrogate model NRSur7dq4 [30]. The former signal model does not include the effects of the precession and higher-order modes to calculate the waveforms, while the latter model directly interpolates the numerical relativity solutions and contains the mentioned effects. For correct comparison with the cWB point estimate, the simulated signals from both models are treated the same way as the real GW signal: Simulated signals are selected randomly from the LALInference posterior distribution, injected into the data (at random times in the time interval of $4096 \mathrm{~s}$ around the GW190521 event), and analyzed with cWB. Then we use the cWB reconstruction to produce the off-source point estimate for each of the simulated events. These different off-source simulations take into account the fluctuations of the real detector noise, cWB reconstruction errors, and the variability of the signal model in the LALInference posterior distribution. The off-source simulations are synchronized in time with GW190521 and used for the construction of confidence intervals showing the expected spread of the modeled signal amplitudes as seen by $\mathrm{cWB}$, which can be directly compared with the cWB point estimate for GW190521. The result is shown in Fig. 5 where the $90 \%$ confidence intervals are calculated from whitened waveforms of approximately 1400 simulated events (it excludes a few percent of events that are not detected). They display that with $90 \%$ probability the local amplitudes of the GW190521 signal filtered by cWB are located within these boundaries. The cWB reconstruction of GW190521 comprises about 11 degrees of freedom (the effective 


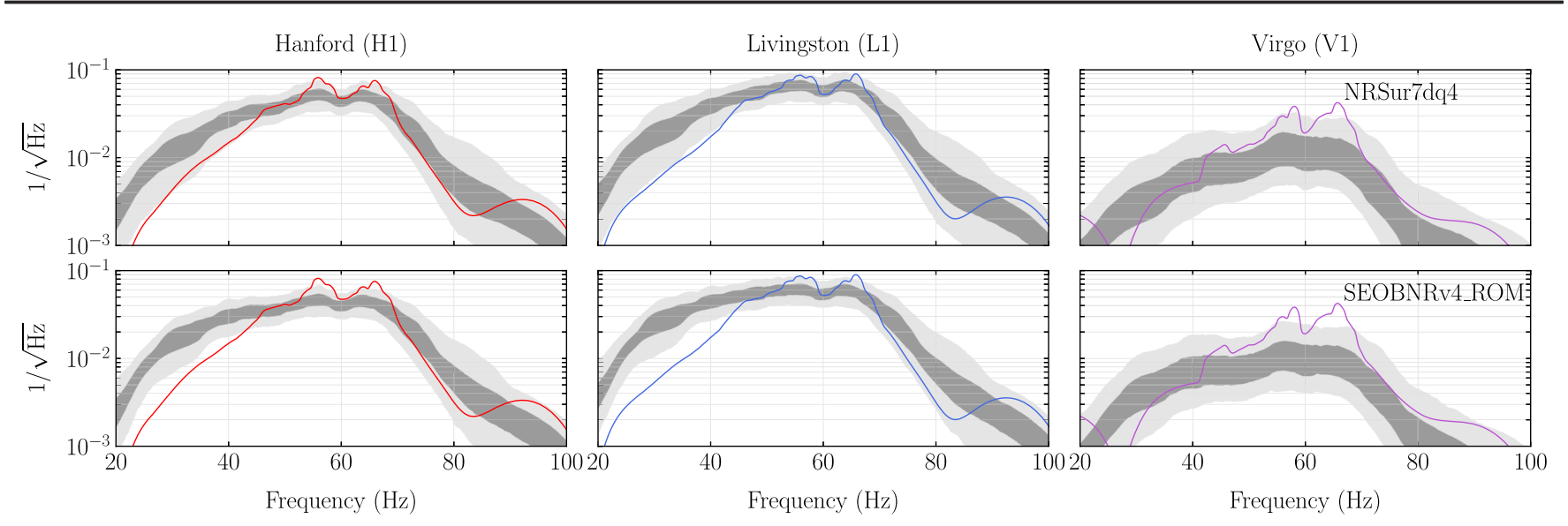

FIG. 6. Visualization of the consistency of the signal-agnostic measurement by cWB with two alternative models in the frequency domain: upper panel, NRSur7dq4 model; lower panel, SEOBNRV4_ROM model. The colored curves are the whitened waveform spectra for GW190521 as reconstructed by cWB for the LIGO Hanford (red), LIGO Livingston (blue), and Virgo (violet); they are a direct measurement from the data and do not change from the upper to the lower panels. The dark shaded belts are the 50\% confidence intervals per frequency bin showing the variation of the model spectral amplitudes. The light shaded belts are the $90 \%$ confidence intervals.

number of independent wavelets), so the confidence intervals for the contiguous time values are correlated. The local deviations outside of the $90 \%$ interval visible in the top plots for the NRSur7dq4 models are negligible. However, the SEOBNOV4_ROM model appears to have larger deviation at the beginning of the GW190521 signal. The difference between the two models is also observed in the frequency domain. Figure 6 shows $90 \%$ confidence intervals built from the Fourier representation of the whitened off-source reconstructions of simulated events. This analysis highlights more discrepancy between the NRSur7dq4 and SEOBNRV4_ROM models than in the time domain. In particular, at lower frequency the NRSur7dq4 model shows a deficit of energy in comparison with the spin-aligned SEOBBNRV4_ROM model, demonstrating a better agreement with the cWB reconstruction of GW190521. Such a deficit of energy before the merger is expected for signals with orbital precession predicted by the NRSur7dq4 model or signals with large eccentricity [37].

\section{A. Waveform overlap}

The time and frequency confidence intervals are a convenient tool to identify local waveform differences between the cWB reconstruction of GW190521 and the signal model. However, they do not provide a statistical measure for disagreement between a given model and the GW190521 signal. To quantify the consistency between different waveforms, we use the waveform overlap, or the match [5,38], between the cWB point estimate $\mathbf{w}=$ $\left\{w_{k}(t)\right\}$ and a selected whitened waveform from a given signal model $\mathbf{h}=\left\{h_{k}(t)\right\}$ :

$$
\mathrm{O}(\mathbf{w}, \mathbf{h})=\frac{(\mathbf{w} \mid \mathbf{h})}{\sqrt{(\mathbf{w} \mid \mathbf{w})} \sqrt{(\mathbf{h} \mid \mathbf{h})}}
$$

The scalar product (.|.) is defined in the time domain as

$$
(\mathbf{w} \mid \mathbf{h})=\sum_{k} \int_{t_{1}}^{t_{2}} w_{k}(t) h_{k}(t) d t
$$

where the index $k$ running over the detectors and $\left[t_{1}, t_{2}\right]$ is the time range of the reconstructed event. First, we calculate the distribution of $\mathrm{O}_{0}\left(\mathbf{w}_{\mathbf{i}}, \mathbf{h}_{\mathbf{i}}\right)$ for the off-source injections, where the waveform $\mathbf{h}_{\mathbf{i}}$ is drawn randomly from the posterior distribution, and the $\mathbf{w}_{\mathbf{i}}$ is the cWB point estimate of $\mathbf{h}_{\mathbf{i}}$. The $\mathrm{O}_{0}$ gives us the null distribution expected for a given signal model accounting for systematic uncertainties due to non-Gaussian detector noise and the cWB reconstruction. Second, we calculate the overlap $\mathrm{O}\left(\mathbf{W}, \mathbf{h}_{\mathbf{p}}\right)$ where $\mathbf{W}$ is the on-source cWB reconstruction of GW190521, and $\mathbf{h}_{\mathbf{p}}$ is a proxy of the true GW190521 signal selected from the posterior distribution. If the model accurately describes the GW190521 event, we expect that the overlap $\mathrm{O}\left(\mathbf{W}, \mathbf{h}_{\mathbf{p}}\right)$ falls within the null distribution, which can be characterized with the $\mathrm{p}$ value - the fraction of entries in the null distribution with the overlap below $\mathrm{O}\left(\mathbf{W}, \mathbf{h}_{\mathbf{p}}\right)$. The left panel in Fig. 7 shows the null distribution and the overlap with the maximum likelihood (max-L) template used as the proxy $\mathbf{h}_{\mathbf{p}}$ for the GW190521 signal evaluated for both signal models. The max-L overlaps are 0.89 and 0.85 , while the p values are $7.9 \%$ and $1.0 \%$ for the NRSur7dq4 and SEOBNRV4_ROM models, respectively. In agreement with Figs. 5-7 illustrates that the latter signal model is less consistent with the GW190521 event. However, the relatively large $\mathrm{p}$ value of $1 \%$ does not allow us confident exclusion of SEOBNRV4_ROM as a model for GW190521.

The assumption of the max- $\mathrm{L}$ waveform being the best representation of GW190521 is viable but not exclusive. 

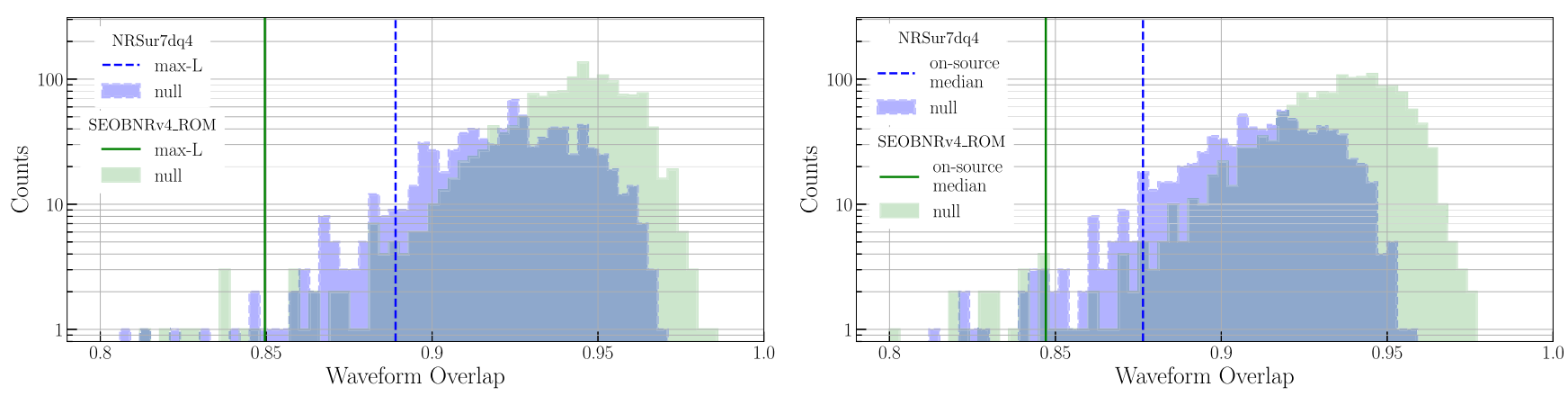

FIG. 7. The quantitative comparison of whether the signal models are consistent with cWB reconstruction of GW190521. The plots show the off-source (null) distributions and calculated waveform overlaps for GW190521. Left panel: the max-L is assumed to be a representative waveform for GW190521. Right panel: any posterior waveform is assumed to be a valid representation of GW190521. In both cases, the p values are $7.9 \%$ and $1.0 \%$ for NRSur7dq4 and SEOBNRV4_ROM, respectively. It indicates that SEOBNRV4_ROM is less consistent with GW190521, but its relatively large p value does not allow us to confidently exclude this model.

The maximum posterior waveform could also be used instead of the max-L. In addition to this ambiguity, a selection of any specific proxy waveform $\mathbf{h}_{\mathbf{p}}$ introduces a bias in the calculation of the $\mathrm{p}$ value. To eliminate this bias, for each off-source injection $\mathbf{h}_{\mathbf{i}}$, we should run the LALInference parameter estimation to find the corresponding proxy waveform, which should be used instead of $\mathbf{h}_{\mathbf{i}}$ for the calculation of the null distribution. This is not practically feasible due to the high computational cost of the LALInference analysis. Instead, we modify the statistical procedure in order to reduce the selection bias. In general, for a correct model, any posterior waveform could be a fair proxy $\mathbf{h}_{\mathbf{p}}$ for the GW190521 signal. Therefore, we calculate the on-source overlap distribution $\mathrm{O}\left(\mathbf{W}, \mathbf{h}_{\mathbf{i}}\right)$ by randomly drawing around $1000 \mathbf{h}_{\mathbf{i}}$ from the posterior distribution. Instead of selecting a specific $\mathrm{O}\left(\mathbf{W}, \mathbf{h}_{\mathbf{p}}\right)$ overlap, we calculate the median of the on-source distribution. Similarly, a set of distributions $\mathrm{O}\left(\mathbf{w}_{\mathbf{j}}, \mathbf{h}_{\mathbf{i}}\right)$ can be calculated for a randomly selected $1000 \mathrm{cWB}$ point estimate $\mathbf{w}_{\mathbf{j}}$ from the off-source injections. By calculating the median of each $\mathrm{O}\left(\mathbf{w}_{\mathbf{j}}, \mathbf{h}_{\mathbf{i}}\right)$ distribution, we can build a new null distribution for the median of $\mathrm{O}\left(\mathbf{W}, \mathbf{h}_{\mathbf{i}}\right)$. The right panel of Fig. 7 shows these null distributions and the onsource median overlap values of 0.88 and 0.85 for the NRSur7dq4 and SEOBNRV4_ROM models, respectively. The corresponding $p$ values are $7.9 \%$ and $1.0 \%$. These numbers are similar to the results obtained from the analysis using the max-L waveform. Nevertheless, the comparison of the entire posterior distribution with the corresponding offsource null distributions provides a more robust test of the GW190521 models.

\section{SUMMARY}

The cWB searches for GW transients demonstrated confident detection of binary black holes, including the first gravitational-wave signal GW150914 detected in the O1 run [9], the heaviest BBH system GW170729 detected in the $\mathrm{O} 2$ run, together with the other detections from the first two observing runs of Advanced LIGO and Advanced Virgo [10]. The observation of GW190521, the first confident detection of an IMBH by cWB, is yet another demonstration of its capabilities to discover new GW sources. The cWB detection in low latency provided a robust reconstruction of GW190521 including a sky map that was in agreement with the template-based searches. The offline cWB analysis provided a confident detection of GW190521 with the false-alarm rate of 1 per 4900 years. The estimated rate of events similar to GW190521 $\left(0.13_{-0.11}^{+0.3} \mathrm{Gpc}^{-3} \mathrm{yr}^{-1}\right.$ [24] $)$ is below the previously derived upper limits. The waveform analysis presented in the paper demonstrates the agreement between cWB and LALInference reconstructions for different signal models. The consistency between the signal models and cWB reconstruction is described by $\mathrm{p}$ values, which are $7.9 \%$ and $1.0 \%$ for the NRSur7dq4 and SEOBNRV4_ROM models, respectively. While the $p$ value of NRSur7dq4 shows that this model is consistent with the cWB reconstruction of GW190521, the relatively large $\mathrm{p}$ value for the SEOBNRV4_ROM signal model does not allow us to confidently exclude this model.

The anticipated sensitivity improvements of the GW instruments at low frequency [23] can lead to detections of more IMBH sources. These sources could be formed dynamically and may have waveforms not covered by existing templates. The template-independent search has the potential to play an important role in the detection of such signals and help us to explore the physical properties of IMBH sources in a wide range of masses.

\section{ACKNOWLEDGMENTS}

This research has made use of data, software, and/or web tools obtained from the Gravitational Wave Open Science Center, a service of LIGO Laboratory, the LIGO Scientific Collaboration, and the Virgo Collaboration. The work by S. K. was supported by National Science Foundation Grant No. PHY 1806165. We gratefully acknowledge the support of LIGO and Virgo for provision of computational 
resources. I. B. acknowledges support by the National Science Foundation under Grant No. PHY 1911796, the Alfred P. Sloan Foundation, and by the University of Florida.

\section{APPENDIX A: COHERENT WAVEBURST}

cWB is a search algorithm for detection and reconstruction of GW transient signals operating without a specific waveform model. cWB searches for a coincident signal power in multiple detectors for signals with duration up to a few seconds. The analysis is performed in the wavelet domain [34] on data normalized (whitened) by the amplitude spectral density of the detector noise. The cWB selects wavelets with amplitudes above the fluctuations of the detector noise and groups them into clusters. For clusters correlated in multiple detectors, cWB identifies coherent events and reconstructs the source sky location and signal waveforms with the constrained maximum likelihood method [15].

The cWB detection statistic is based on the coherent network energy $E_{\mathrm{c}}$ obtained by cross-correlating data in different detectors; $\sqrt{E_{\mathrm{c}}}$ is the estimator of the coherent network SNR [15]. The agreement between GW data and $\mathrm{CWB}$ reconstruction is characterized by a chi-squared statistic $\chi^{2}=E_{\mathrm{n}} / N_{\mathrm{df}}$, where $E_{\mathrm{n}}$ is the residual energy estimated after the reconstructed waveforms are subtracted from the whitened data, and $N_{\mathrm{df}}$ is the number of independent wavelet amplitudes describing the event. GW190521 is identified in LIGO data with $\chi^{2}=0.68$, showing no evidence for residual energy inconsistent with Gaussian noise. The cWB detection statistic is $\eta_{\mathrm{c}}=\sqrt{E_{\mathrm{c}} / \max \left(\chi^{2}, 1\right)}$, where the $\chi^{2}$ correction improves the robustness of the pipeline against nonGaussian detector noise. Events with $\eta_{\mathrm{c}}>5$ are stored by the pipeline for further processing.

To improve the robustness of the algorithm against nonstationary detector noise (glitches) and reduce the rate of false alarms, cWB uses signal-independent vetoes. The primary veto cuts are on the network correlation coefficient $c_{\mathrm{c}}=E_{\mathrm{c}} /\left(E_{\mathrm{c}}+E_{\mathrm{n}}\right)$ and the $\chi^{2}$. For a GW signal, the expected values of both statistics are close to unity, and the candidate events with $c_{\mathrm{c}}<0.7$ and $\chi^{2}>2.5$ are rejected as potential glitches.

\section{APPENDIX B: MITIGATION OF NONSTATIONARY DETECTOR NOISE}

The analysis of the early $\mathrm{O} 3$ data revealed several dominant sources of nonstationary noise (glitches). The dominant sources of this noise are the low-frequency glitches below $50 \mathrm{~Hz}$ due to anthropogenic ground motion and the short-duration (blip) glitches in the frequency band $50-300 \mathrm{~Hz}[39,40]$. The anthropogenic ground motion in LIGO Livingston (LLO) produced the scattered light noise below $50 \mathrm{~Hz}$ [5] appearing as a periodic sequence of
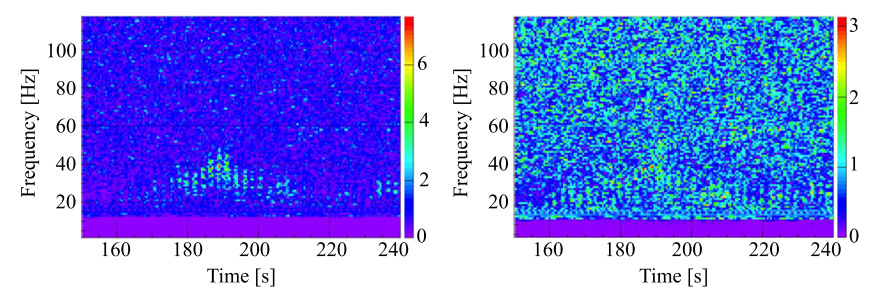

FIG. 8. Time-frequency Wilson-Daubechiers-Meyer wavelet decomposition of a segment of LLO data: left (right) is before (after) application of the LPE filter. The excess power due to anthropogenic ground motion is reduced.

short-duration transients. A similar type of noise is also observed in the LIGO Hanford (LHO) detector, but at a significantly lower rate. The left panel of Fig. 8 shows an example of scatter light glitches in the time-frequency map of the LLO data segment. This noise is highly periodic with the two characteristic times of 2.35 and $0.235 \mathrm{~s}$. It can be corrected with the linear predictor error filter (LPE) [41] as shown in the right panel of Fig. 8. The correction of the wavelet data was performed in the frequency band 16-48 $\mathrm{Hz}$ and does not affect the detection and reconstruction of signals at higher frequency. No scattered light glitches were observed at the time of GW190521, which has a peak frequency of $58 \mathrm{~Hz}$. The application of the LPE filter significantly reduces the rate of the scattered light glitches and improves the detection of IMBH binary signals expected at low frequencies.

The short noise transients are primarily blip glitches present in all observing runs and their origin is yet unknown [39,40]. The properties of blip glitches have been extensively studied in the previous observing runs [42], and to remove them, we apply a similar method used in the previous cWB searches $[10,22]$. The waveforms are typically time symmetric, with less than one cycle, and without a clear frequency evolution. The duration is usually of the order of $O(10) \mathrm{ms}$ and bandwidth $O(100) \mathrm{Hz}$. Figure 9 shows an example of a blip glitch.

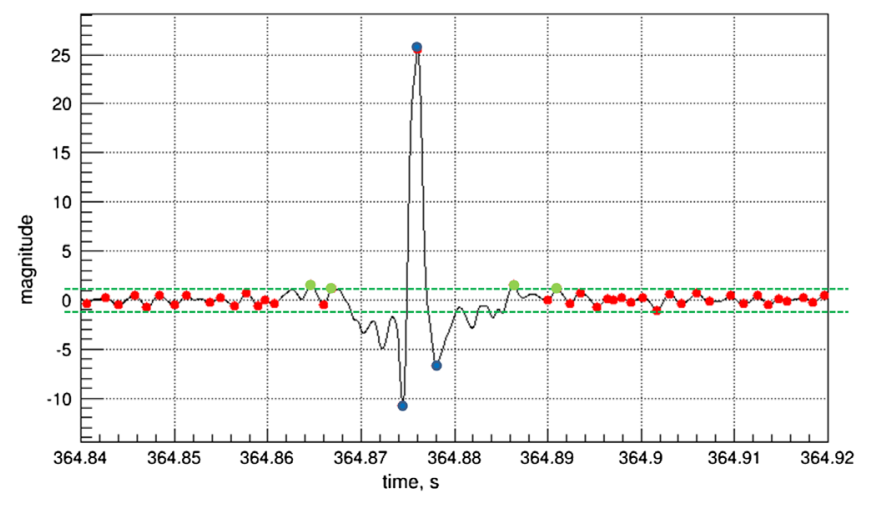

FIG. 9. An example of a blip glitch. The characteristic waveforms of blip glitches allow us to distinguish them from gravitational waves and other sources of noise as described in the text. 
TABLE I. Possible detection cases and corresponding trial factors. For example, in case 5, an event is identified in both search configurations, but the peak frequencies are reconstructed in different bands, so the smaller false-alarm rate from the two configurations multiplied by a trial factor of 2 defines the event's significance. The calculation of GW190521 significance is given by case 3 .

\begin{tabular}{lccccccc}
\hline \hline Case & 1 & 2 & 3 & 4 & 5 & 6 & 7 \\
\hline IMBH configuration & IMBH,BBH & - & IMBH & - & IMBH & BBH & BBH \\
BBH configuration & - & IMBH,BBH & - & BBH & BBH & IMBH & - \\
False-alarm rate & IMBH & BBH & IMBH & BBH & & min $(\mathrm{BBH}, \mathrm{IMBH}) \times 2$ & IMBH \\
\hline \hline
\end{tabular}

The characteristic waveform of blip glitches allows their simple classification as follows. The energy of the data samples with the largest magnitude $\left(A_{m}\right)$ and two peak amplitudes around it (blue dots) is denoted as $E_{0}$. The remaining samples with the amplitudes outside of the thresholds $\pm A_{m} / 7.6$ (green dots) have the energy denoted as $E_{1}$. The ratio $Q=E_{1} / E_{0}$ is calculated for each detector used to reconstruct an event, and the smallest value of $Q$ out of all detectors may indicate the presence of a blip glitch. Events with $Q<0.1$ are removed from further analysis. GW190521 with a $Q$ value of 0.55 passed this criteria.

\section{APPENDIX C: SEARCH CONFIGURATIONS}

A generic search for binary systems covers a large parameter space. It is not possible to design a search optimized to all binaries because the frequency content of their GW signals can vary significantly. In general, $\mathrm{GW}$ signals have the peak frequency $f_{\text {peak }} \propto 1 / M_{\text {tot }}$, where $M_{\text {tot }}$ is the binary total mass in the detector frame. Lighter binaries with $M_{\text {tot }} \approx 2 M_{\odot}$ merge at high frequency, and their GW signal may be observable in the detector data for hundreds of seconds. Heavier systems with $M_{\text {tot }}>100 M_{\odot}$ merge at lower frequencies, and their detected signals are less than a second long. Therefore, a generic search for binary systems is split into the two search configurations. One configuration focuses on the IMBH binaries $\left(f_{\text {peak }}<80 \mathrm{~Hz}\right)$, while the other configuration is used for the detection of stellar mass BBHs $\left(f_{\text {peak }}>80 \mathrm{~Hz}\right)$. Because the detector noise and glitches vary significantly in these two frequency bands, the searches require different selection cuts.

The two configurations are not disjoint, and trial factors need to be considered to calculate the event's significance, and they are determined by the reconstructed peak frequencies. Table I summarizes all cases when a binary system is detected either by the BBH or IMBH search configuration. For example, if the event is detected in both search configurations with the peak frequencies above $80 \mathrm{~Hz}$, then the BBH search is selected. and the significance of the event is not penalized by the trial factor. In contrast, if the peak frequencies of the detected signals are reconstructed in different frequency bands, then the smaller FAR is used for the calculation of the event's significance, and it is multiplied by a trial factor of 2 .
[1] J. Aasi et al. (LIGO Scientific Collaboration), Classical Quantum Gravity 32, 074001 (2015).

[2] F. Acernese et al. (VIRGO Collaboration), Classical Quantum Gravity 32, 024001 (2015).

[3] B. P. Abbott et al. (LIGO Scientific and Virgo Collaborations), Phys. Rev. Lett. 892, L3 (2020).

[4] B. P. Abbott et al. (LIGO Scientific and Virgo Collaborations), Phys. Rev. D 102, 043015 (2020).

[5] B. P. Abbott et al. (LIGO Scientific and Virgo Collaborations), Phys. Rev. Lett. 125, 101102 (2020).

[6] Gravitational-Wave Candidate Event Database, LIGO/Virgo public alerts, https://gracedb.ligo.org/superevents/public/ O3/ (2020).

[7] T. Ebisuzaki, J. Makino, T. G. Tsuru, Y. Funato, S. F. Portegies Zwart, P. Hut, S. McMillan, S. Matsushita, H. Matsumoto, and R. Kawabe, Astrophys. J. 562, L19 (2001).

[8] M. Mezcua, Int. J. Mod. Phys. D 26, 1730021 (2017).

[9] B. P. Abbott et al. (LIGO Scientific and Virgo Collaborations), Phys. Rev. Lett. 116, 061102 (2016).
[10] B. P. Abbott et al. (LIGO Scientific and Virgo Collaborations), Phys. Rev. X 9, 031040 (2019).

[11] M. Spera and M. Mapelli, Mon. Not. R. Astron. Soc. 470, 4739 (2017).

[12] S. E. Woosley, Astrophys. J. 836, 244 (2017).

[13] N. Giacobbo, M. Mapelli, and M. Spera, Mon. Not. R. Astron. Soc. 474, 2959 (2018).

[14] K. Belczynski et al., Astron. Astrophys. 594, A97 (2016).

[15] S. Klimenko et al., Phys. Rev. D 93, 042004 (2016).

[16] J. Calderón Bustillo, F. Salemi, T. Dal Canton, and K. P. Jani, Phys. Rev. D 97, 024016 (2018).

[17] K. Chandra, V. Gayathri, J. C. Bustillo, and A. Pai, Phys. Rev. D 102, 044035 (2020).

[18] J. Abadie et al. (LIGO Scientific and VIRGO Collaborations), Phys. Rev. D 85, 102004 (2012).

[19] J. Aasi et al., Phys. Rev. D 89, 122003 (2014).

[20] J. Aasi et al., Phys. Rev. D 89, 102006 (2014).

[21] B. P. Abbott et al. (LIGO Scientific and Virgo Collaborations), Phys. Rev. D 96, 022001 (2017). 
[22] B. P. Abbott et al. (LIGO Scientific and Virgo Collaborations), Phys. Rev. D 100, 064064 (2019).

[23] B. P. Abbott et al. (KAGRA, LIGO Scientific, and Virgo Collaborations), Living Rev. Relativity 19, 1 (2016).

[24] B. P. Abbott et al., Astrophys. J. 900, L13 (2020).

[25] J. Veitch, V. Raymond, B. Farr et al., Phys. Rev. D 91, 042003 (2015).

[26] G. Mo et al. (LIGO Scientific and Virgo Collaborations), Report No. 24621, 2019.

[27] A. Nitz et al., GWASTRO/PYCBC v1.15.3, 2020.

[28] L. P. Singer and L. Price, Phys. Rev. D 93, 024013 (2016).

[29] A. Bohé et al., Phys. Rev. D 95, 044028 (2017).

[30] V. Varma, S. E. Field, M. A. Scheel, J. Blackman, D. Gerosa, L. C. Stein, L. E. Kidder, and H. P. Pfeiffer, Phys. Rev. Research 1, 033015 (2019).

[31] G. Ashton et al., Astrophys. J. Suppl. Ser. 241, 27 (2019).

[32] J. Lange et al., Phys. Rev. D 96, 104041 (2017).

[33] N. J. Cornish and T.B. Littenberg, Classical Quantum Gravity 32, 135012 (2015).
[34] V. Necula, S. Klimenko, and G. Mitselmakher, J. Phys. Conf. Ser. 363, 012032 (2012).

[35] S. Ossokine et al., Phys. Rev. D 102, 044055 (2020).

[36] S. Babak, A. Taracchini, and A. Buonanno, Phys. Rev. D 95, 024010 (2017).

[37] J. Calderón Bustillo, N. Sanchis-Gual, A. Torres-Forné, and J. A. Font, arXiv:2009.01066.

[38] F. Salemi, E. Milotti, G. A. Prodi, G. Vedovato, C. Lazzaro, S. Tiwari, S. Vinciguerra, M. Drago, and S. Klimenko, Phys. Rev. D 100, 042003 (2019).

[39] B. P. Abbott et al. (LIGO Scientific and Virgo Collaborations), Classical Quantum Gravity 35, 065010 (2018).

[40] M. Cabero et al., Classical Quantum Gravity 36, 155010 (2019).

[41] V. Tiwari, M. Drago, V. Frolov, S. Klimenko, G. Mitselmakher, V. Necula, G. Prodi, V. Re, F. Salemi, G. Vedovato, and I. Yakushin, Classical Quantum Gravity 32, 165014 (2015).

[42] B. P. Abbott, R. Abbott, T. D. Abbott et al. (LIGO Scientific and Virgo Collaborations), Classical Quantum Gravity 33, 134001 (2016). 